\title{
Resistencias epistémico-políticas frente a la privatización de las semillas y los saberes colectivos
}

\author{
Epistemic and Political Struggles against the Privatization \\ of Seeds and Collective Knowledges
}

DOI: $10.22380 / 2539472 X .798$

\author{
Nathalia Hernández Vidal ${ }^{*}$ \\ Oxford College of Emory, Estados Unidos \\ Laura Gutiérrez Escobar"* \\ Universidad del Rosario, Bogotá
}

\begin{abstract}
RESUMEN
En un contexto de libre comercio y privatización de los bienes comunes, analizamos la producción de conocimiento sobre semillas nativas y criollas en la Red de Semillas Libres de Colombia, en dos dimensiones: 1) otras formas de saber y relacionarse con las semillas; 2) los mecanismos por los cuales estas formas de saber han sido o no incluidas en la política pública. Partiendo de estudios críticos sobre ciencia y tecnología y decolonialidad, argumentamos que existe un conflicto entre dos concepciones, una derivada de discursos y prácticas del desarrollo, en los que las semillas son el capital de la industria agrobiotecnológica y los saberes asociados con su cultivo y preservación se privatizan. Y otra donde semilla, territorio y saber se entretejen como bien común.
\end{abstract}

Palabras claves: Red de Semillas Libres de Colombia, decolonialidad, bienes comunes, privatización.

\begin{abstract}
$\overline{\text { ABSTRACT }}$
Framed in a context of free trade policies and the privatization of the commons, we analyze the production of knowledge about 'traditional' seeds in the Colombian Network of Free Seeds, on two dimensions: (1) other ways of knowing and relating to seeds; (2) mechanisms by which these forms of know-how have been included or excluded from public policy. Drawing from critical studies on science and technology and decoloniality, we argue that there is a conflict between two forms of conceiving seeds. One, born out of discourses and practices of development, where seeds are the capital of the agricultural biotechnology industry and the knowledges on how to preserve and grow them are privatized. Another in which seeds, territory, and knowledge interweave as a commons.
\end{abstract}

Keywords: Colombian Network of Free Seeds, decoloniality, commons, privatization.

Filósofa y candidata a doctora en Sociología de Loyola University Chicago. nhernandezvidal@ luc.edu / https://orcid.org/0000-0001-5529-4083

Doctora en Antropología de la University of North Carolina, Chapel Hill. Profesora de la Universidad del Rosario e investigadora posdoctoral de la Universidad Nacional de Colombia. laurittag@yahoo.com / https://orcid.org/0000-0002-2161-4031 


\section{Custodiando las semillas en Colombia'}

Yo soy guardiana de semillas. Yo diría que un guardián de semillas es la persona que asume la responsabilidad de proteger las semillas nativas, de reproducirlas, de distribuirlas [...] Pero hace también un ejercicio de soberanía alimentaria, de soberanía territorial; es un ejercicio de liberación, de rebeldía y definitivamente, un ejercicio espiritual. Velma y Hernando, guardianes de semillas, $2010^{2}$

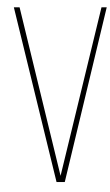

elma y Hernando pertenecen a la Red de Semillas Libres de Colombia (RSLC). Viven en uno de los territorios libres de transgénicos (TLT) del país ubicado en el resguardo indígena Embera-Chamí de Cañamomo-Lomaprieta, en el departamento de Caldas. Los resguardos son territorios indígenas colectivos e inalienables, algunos de origen colonial, reconocidos por la Constitución colombiana de 1991 como parte de los derechos de los pueblos indígenas al autogobierno dentro de un marco político multiétnico y pluricultural. En su resguardo, una de las labores principales es cuidar las semillas nativas y criollas usando saberes comunitarios y enseñanzas de la agricultura agroecológica. Como sus palabras lo señalan, Velma y Hernando se piensan como guardianes de semillas, esto es, como personas que han asumido la responsabilidad de velar por el bienestar de esas semillas y de los espacios que las acogen.

Dada la centralidad de las semillas en este artículo, consideramos importante aclarar la terminología que usamos para referirnos a ellas. Las semillas nativas - también llamadas originarias - son aquellas cuyo centro de origen se encuentra en la misma región en la que son cultivadas. Las semillas criollas no son necesariamente originarias de una región, pero los agricultores las han adaptado al clima y a las condiciones ambientales de sus huertas y parcelas. En la expresión semillas criollas también incluimos las semillas creolizadas o semillas certificadas/mejoradas científicamente que fueron adaptadas por los agricultores a las condiciones ecosistémicas de sus huertas, parcelas y territorios, así como cruzadas intencional o accidentalmente con variedades criollas.

Queremos agradecer a los evaluadores anónimos por sus comentarios a este artículo, así como a los miembros de la Red de Semillas Libres de Colombia por sus enseñanzas durante todos estos años de trabajo. También a Natalia Pérez y Birgit Hoinle por su cuidadosa lectura de una versión previa de este artículo.

Con el fin de proteger a los participantes, los nombres mencionados en este artículo son ficticios, con excepción de Velma Echavarría y Hernando Escobar, quienes expresaron su consentimiento para ser visibilizados. 
Velma y Hernando hacen parte de una tradición que ha existido desde siempre en las comunidades rurales alrededor del mundo, pero que en los últimos veinte años se ha estructurado, sistematizado y visibilizado mucho más. Algunos sociólogos como Kloppenburg (2005) y Kinchy (2012) afirman que el activismo alrededor de las semillas en décadas recientes es producto, por un lado, de la revolución verde ${ }^{3} \mathrm{y}$, por otro, de su extensión a través de la agrobiotecnología (biotecnología utilizada en agricultura) y del surgimiento de empresas transnacionales biotecnológicas que concentran los mercados. Nos referimos a tres empresas que controlan el mercado mundial de semillas e insumos químicos: Bayer, que al adquirir Monsanto pasó a controlar la cuarta parte de este mercado; Dow, que adquirió Dupont; y ChemChina (la única empresa estatal) que adquirió Syngenta. El control de los mercados implica tanto el control de las semillas como del aparato legal de derechos de propiedad intelectual (DPI) y de certificación de semillas. Los conflictos generados por el uso, desarrollo y comercialización de semillas a partir de la expansión del poder y capital de las empresas transnacionales y de la implementación de este aparato tecnolegal ${ }^{4}$ han sido llamados conflictos por las semillas (Kloppenburg 2005).

No obstante, dada la heterogeneidad de territorios en los que encontramos este tipo de conflictos, es importante preguntar: ¿cuál es la forma que toman los conflictos por las semillas en el contexto colombiano? Más aún, ¿qué es exactamente lo que está en conflicto: el uso, el acceso o el conocimiento? Estas son las preguntas generales que guían este artículo.

Argumentamos que lo que está en conflicto es la existencia de dos formas muy distintas de concebir las semillas: la primera, derivada de discursos y prácticas del desarrollo (Escobar 2011), de las cuales la revolución verde es uno de los ejemplos más claros. Bajo esta óptica, las semillas se vuelven el capital de la industria agrobiotecnológica y los saberes asociados con su cultivo y preservación se privatizan. La segunda, perteneciente a un universo en el cual semilla, territorio y saber se entretejen para concebir y usar la semilla como bien común.

Con el fin de desarrollar nuestro argumento, abrimos la siguiente sección con dos apartados: uno metodológico y otro teórico. En seguida, mostramos cómo,

3 La revolución verde es un modelo de desarrollo agrícola implementado desde la década de los 1950 en los llamados países del "tercer mundo" y basado en la siembra extensiva de un solo producto (monocultivo). En este modelo, el uso de agroquímicos es fundamental para garantizar la sobrevivencia y productividad de los cultivos.

4 Jasanoff (2013) y Parthasarathy (2017) han enfatizado que la conjunción entre los elementos tecnocientíficos y legales requeridos para que la industria biotecnológica funcione puede pensarse como un aparato tecnolegal. En adelante usaremos dicho término para referirnos a tal ensamblaje. 
en el desarrollo de los conflictos por las semillas en Colombia, los saberes de los guardianes ${ }^{5}$ de semillas se han convertido en uno de los principales bastiones de resistencia. Luego, presentamos algunos de los ejes que componen los conflictos por las semillas en Colombia y las estrategias que han elegido los guardianes en el país para el cuidado y protección de las semillas nativas y criollas. Cerramos con algunas consideraciones acerca de los retos que enfrentan las redes de guardianes de semillas y la RSLC.

\section{Una etnografía de las semillas}

Este artículo está basado en nuestras investigaciones doctorales 6 . Laura Gutiérrez realizó su trabajo de campo entre el 2013 y el 2014 con guardianes de semillas de los resguardos Embera-Chamí de Riosucio, Caldas, vinculados tanto a los programas de soberanía alimentaria de los cabildos ${ }^{7}$ y apoyados por la RSLC, como a una organización agroecológica de base llamada Asproinca ${ }^{8}$. Se utilizaron diversas metodologías cualitativas, incluyendo talleres para discutir los DPI sobre material vegetal, así como observación participante y entrevistas semiestructuradas en reuniones, eventos académicos y mercados campesinos, a escala local, regional y nacional. También se emplearon matrices de biodiversidad (Nazarea 1995, 113-116) para recolectar información sobre la historia y características de semillas/plantas, incluyendo usos medicinales, propiedades agronómicas/ nutritivas y formas de cultivo, almacenamiento, comercialización y consumo.

El trabajo de campo de Nathalia Hernández Vidal fue realizado durante un periodo de tres años en distintas etapas que incluyeron participación en reuniones, encuentros regionales y nacionales, y festivales de comida de la RSLC en Bogotá, Riosucio, Buga, Zipaquirá, Coyaima, La Calera y Pasto. Además de la

5 Si bien las autoras adscriben a un lenguaje inclusivo con perspectiva de género, debido a que la RCA se rige por las normas de la Real Academia Española, en este artículo se conserva el uso genérico del masculino.

6 Laura Gutiérrez Escobar escribió su tesis doctoral sobre los conflictos por las semillas en Colombia desde la perspectiva de la ecología y la ontología políticas. Nathalia Hernández Vidal actualmente escribe su disertación acerca de los procesos de resistencia a la agrobiotecnología y los regímenes de propiedad intelectual llevados a cabo por la Red de Semillas Libres de Colombia.

7 Los cabildos son las formas de gobierno autónomo indígena que rigen en los resguardos y que son garantizados por la Constitución de 1991. 
observación participante, se realizaron entrevistas etnográficas y semiestructuradas. Finalmente, fueron revisados documentos oficiales y de la RSLC en sus distintas formas, ya fueran folletos, escritos jurídicos, comunicados de prensa, entre otros.

\section{Gobernanza y dimensiones epistémicas de los conflictos por semillas}

El debate y la movilización social relacionados con los conflictos de semillas se han concentrado en exigir el etiquetado de productos transgénicos, evaluar los efectos de estos en la salud y el medio ambiente, o estudiar los modelos de gobernabilidad utilizados para su regulación (Kinchy 2012; Parthasarathy 2017). Algunos de estos análisis han vinculado la creación de leyes de semillas y el uso de semillas transgénicas con procesos de acumulación a través del despojo (Harvey 2004) de la diversidad genética. Menos atención se ha prestado al estudio de los tipos de saberes en disputa en relación con los conflictos sobre semillas, vacío que pretendemos abordar en este artículo. Para tal efecto, nos basamos en los estudios sociales de ciencia, tecnología y sociedad (CTS) y el pensamiento decolonial latinoamericano.

Las perspectivas CTS han estado enfocadas en mostrar cómo la ciencia y la tecnología operan en lo político, es decir, en campos de poder a nivel social, económico y normativo, y no por fuera de ellos. Por esta razón, en este artículo usamos la expresión epistémico-político. Teniendo en cuenta este marco, algunos estudios en CTS interrogan los mecanismos bajo los cuales cierto saber es legitimado socialmente e incorporado y traducido en política pública, o invisibilizado y marginalizado. Estos estudios han mostrado que este proceso de legitimación de saberes ocurre a través de nociones como la de experticia (Collins y Evans 2007; Epstein 1996; Jasanoff 2013; Wynne 2003). En este sentido, el conocimiento tecnocientífico se ha considerado como el conocimiento experto por excelencia y ocupa un lugar privilegiado en la formulación e implementación de la política pública en Occidente. Para hablar sobre este proceso, Jasanoff (1990) creó el concepto de ciencia regulatoria, en el cual el personal científico se encarga de construir y sintetizar conocimiento que informe la toma de decisiones, o de crear conocimiento que pueda darle fundamento a una propuesta de política pública.

Así mismo, la ciencia regulatoria no incluye en sus procesos epistémicos conocimiento que no esté certificado como experto y basa sus decisiones en una 
pretensión de objetividad. Dicha objetividad presupone la creación de un conocimiento "libre de valores", abstraído de la cultura o puramente técnico. No obstante, señala Jasanoff, tal objetividad está construida en contextos atravesados por mundos éticos, culturales y sociopolíticos muy específicos que transitan y son transitados por relaciones de poder en las que, por ejemplo, las culturas científicas dominantes suelen tener la última palabra acerca de lo que se considera como los andamiajes (métodos, datos, formas de análisis) y productos de conocimiento legítimos.

En este artículo usamos la perspectiva de Jasanoff en dos sentidos: primero, para analizar cuáles son los conocimientos que se están teniendo en cuenta o se están desplazando a las márgenes a la hora de formular políticas públicas que atañen al mundo rural —en especial a las semillas-. Segundo, para mostrar cuáles son los mundos sociopolíticos, culturales y éticos que atraviesan la construcción de dicha política pública y del conocimiento empleado para direccionarla.

En cuanto al pensamiento decolonial latinoamericano, tomamos específicamente el concepto de colonialidad ${ }^{9}$ cultural (Quijano 2007) que, desde nuestra perspectiva, es una forma de dominación epistémico-política relacionada con el problema de la experticia y de los procesos de inclusión/exclusión del conocimiento en la producción de política pública. Este concepto, que denota la relación de dominación colonial del mundo blanco/europeo sobre las cosmovisiones de los pueblos colonizados, hace énfasis en cómo esa relación es producto de la represión de "[...] modos de conocer, de producir conocimiento, de producir perspectivas, imágenes [...]" (Quijano 2007, 12). En este escrito nos preguntamos si en los conflictos sobre semillas en Colombia puede detectarse un proceso de colonialidad cultural y un proceso paralelo de resistencia o, incluso, de "desmonte". En lo que sigue nos ocupamos de explicar el trabajo de la RSLC y delimitar el contexto político, económico y legal en el cual se enmarcan los conflictos por las semillas en Colombia.

9 Dentro del pensamiento decolonial se establece una diferencia entre colonialismo y colonialidad. Maldonado Torres, por ejemplo, entiende el colonialismo como "una relación política y económica en la cual la soberanía de un pueblo reside en el poder de otro pueblo o nación, lo que constituye a tal nación en un imperio". En contraste con esa relación de soberanía territorial, "la colonialidad se refiere a un patrón de poder [...] a la forma como el trabajo, el conocimiento, la autoridad y las relaciones intersubjetivas se articulan entre sí, a través del mercado capitalista y de la idea de raza. Así pues, aunque el colonialismo precede a la colonialidad, la colonialidad sobrevive al colonialismo" (Maldonado Torres 2007). 


\section{Custodiando semillas en el contexto global}

La RSLC es una red descentralizada de guardianes de semillas, colectivos agroecológicos y comunidades campesinas, indígenas y afrodescendientes, así como de agricultores urbanos, en particular los desplazados por el conflicto armado. ONG ambientalistas y de derechos humanos, grupos de investigación, colectivos artísticos y académicos también hacen parte de ella. La RSLC, creada en el 2012, tiene dos objetivos principales: 1) fortalecer y proteger el uso de semillas nativas y criollas y los conocimientos asociados con ellas; 2) la resistencia civil frente a las leyes de propiedad intelectual y certificación de semillas, y al desarrollo y uso de alimentos y cultivos transgénicos. La RSLC opera en todo el país, pero tiene mayor presencia y capacidad de acción allí donde se encuentran las redes de guardianes de semillas y colectivos agroecológicos más consolidados. Algunas de las regiones más organizadas son Caldas, Risaralda y Antioquia, donde la Red Colombiana de Agricultura Biológica (Recab) y las redes de guardianes de semillas de Riosucio, Caldas, tienen un papel protagónico. En Santander, organizaciones como Fundaexpresión y las Escuelas Agroecológicas son notorias. En el sur del país, en especial en Nariño y Cauca, la Red de Guardianes de Semillas de Vida y en la costa atlántica, la Red Agroecológica del Caribe (Recar) y los guardianes de semillas del pueblo indígena zenú del Resguardo de San Andrés de Sotavento en Córdoba, han contribuido ampliamente al avance y crecimiento de la organización (RSLC y Grupo Semillas 2015).

La RSLC es un caso de estudio interesante porque es una organización que opera no solo ejerciendo resistencia a los DPI, las leyes de comercialización y certificación y las semillas transgénicas, sino también cultivando y circulando conocimientos y formas de conocimiento sobre las semillas, que los mecanismos oficiales establecidos para la generación e implementación de política pública para el mundo rural-agrario no consideran relevantes o legítimos ${ }^{10}$. Nuestro estudio muestra cómo a través del trabajo de mantenimiento, fortalecimiento y ampliación de las redes de guardianes de semillas, y la creación y promoción de

Existen otras iniciativas de conservación de semillas nativas y criollas que ejercen oposición frente a las leyes de comercialización y certificación en otras partes del mundo y con las que la RSL ha establecido relaciones de colaboración y solidaridad. Por ejemplo, la RSL hace parte de la Red por una América Libre de Transgénicos y está en comunicación con Navdanya, una organización india a la que pertenece la reconocida activista y física Vandana Shiva, que promueve la conservación de la biodiversidad, la agroecología y los derechos de los agricultores a través de redes de guardianes de semillas. Igualmente relevante es el African Center for Biodiversity que impulsa estos procesos en África, en especial, frente a la expansión de programas filantrópicos corporativos — como la Fundación Melinda y Bill Gates- que debilitan las iniciativas de base por la soberanía en semillas. 
casas de semillas y escuelas agroecológicas, la RSLC genera conocimiento experto sobre las semillas que amplía la base de biodiversidad de nuestro país y que contribuye a la preservación de saberes y semillas como bienes comunes. Sin embargo, antes de entrar en detalle acerca de cómo sucede esto dentro de la red, es necesario describir mejor el contexto de las leyes de semillas al que nos hemos referido.

\section{Leyes de semillas}

Las leyes de semillas se centran en tres aspectos interrelacionados: los DPI sobre material vegetal — como los derechos de obtentor y patentes-, las certificaciones de calidad y fitosanidad, y las certificaciones de comercialización.

Los DPI, regulados por el Convenio de la Unión para la Protección de las Obtenciones Vegetales (UPOV) de la Organización Mundial del Comercio (OMC), otorgan derechos a aquellos que desarrollan nuevas variedades vegetales sobre su uso, reproducción y comercialización. En la UPOV, los derechos de obtentor se otorgan a una variedad nueva, distinta, uniforme y estable por un término definido y con excepciones para garantizar la reserva del agricultor —o la práctica consuetudinaria de guardar semillas de su propia cosecha-, así como para su uso como recurso genético en la investigación y el desarrollo de nuevas variedades. No obstante, a medida que la industria semillera se ha expandido hasta convertirse en empresas transnacionales de biotecnología que invierten millones de dólares en el desarrollo de variedades certificadas, ya sean transgénicas o no, los DPI sobre material vegetal se han ampliado incluyendo patentes y volviendo los derechos de obtentor más restrictivos para poder garantizar el retorno de estas inversiones multimillonarias.

Tales cambios se han introducido en especial con la versión de 1991 de la UPOV (UPOV91). Las patentes se aplican no solo sobre la semilla, sino también sobre otras partes de las plantas, su material genético y las técnicas para el desarrollo de nuevas variedades. Aún más, la UPOV91 establece que los derechos de obtentor y las patentes se extienden no solo al desarrollo de nuevas variedades, sino a su "descubrimiento", es decir, a las variedades que no estén registradas ante una oficina de propiedad intelectual de la Organización Mundial de Propiedad Intelectual (OMPI). Estos derechos también cobijan aquellas variedades que sean consideradas "esencialmente derivadas” de las variedades protegidas (Góngora-Mera y Motta 2014; Grupo Semillas 2011; Gutiérrez y Fitting 2016).

Elizabeth Bravo (2005, 2014), Vandana Shiva (2001) y otros estudiosos de los conflictos sobre semillas interpretan esta ampliación de los DPI sobre material 
vegetal como un proceso de legalización de la biopiratería - entendida como la apropiación de las semillas nativas y criollas para su explotación comercial sin el conocimiento o consentimiento de los pueblos que las han desarrollado y conservado-, que se convierte en el cercamiento de un bien común. Colombia, como parte de los compromisos del Tratado de Libre Comercio con Estados Unidos, implementó la ampliación de los DPI sobre material vegetal a partir de su adhesión a la UPOV91 (Gobierno Nacional de Colombia 2006).

Esta apropiación, que restringe el uso y acceso de los pueblos a sus semillas, es posible por diversas razones. Primero, dado que en las comunidades rurales las semillas han sido de uso, propiedad y circulación colectiva como bienes comunes, no existen ni la práctica ni la intención de registrar las semillas de sus territorios ante la OMPI. Algunas de las entrevistas realizadas en nuestro trabajo de campo revelan este argumento de forma clara. Por ejemplo, Mariela, en el Segundo Encuentro Nacional de la RSLC en el Resguardo Indígena de CañamomoLomaprieta en el 2015, señalaba que:

Acá para nosotros ha sido muy importante rotar siempre las semillas, compartir lo que tenemos y enseñarnos los unos a los otros. Entonces es muy jodido que vengan luego a decirnos que esas semillas no son nuestras o que no las podemos usar. Mucho menos se nos iba a ocurrir registrarlas. ¿Registrarlas en dónde? ¿Para qué?

Segundo, así las comunidades estuvieran dispuestas a proteger sus semillas con DPI, estas son por definición heterogéneas y cambiantes en la medida en que los agricultores experimentan con ellas en sus campos de cultivo, las intercambian y las adaptan a sus condiciones socioambientales y, por tanto, no cumplen con los requerimientos mencionados para obtener DPI (productividad, pureza, novedad, uniformidad y estabilidad). Tercero, las semillas de la industria son desarrolladas a partir de la inmensa diversidad de semillas que han conservado las comunidades y, por tanto, comparten características que las hacen “esencialmente derivadas” (Grupo Semillas 2011; Gutiérrez y Fitting 2016).

En cuanto a la certificación de semillas, con la expansión de la agricultura industrial y la aplicación del conocimiento científico occidental al desarrollo de semillas -incluyendo la agronomía y la genómica-, se ha construido un consenso sobre las semillas industriales como "mejoradas" frente a las nativas y criollas que se definen como la materia prima genética (Shiva 2001). De esta manera, las semillas criollas y nativas no son objeto de certificación dado que son consideradas como propagadoras de plagas y enfermedades y de baja calidad, especialmente en términos de productividad. Esta noción ha sido defendida en Colombia de manera abierta y pública por servidores del Estado. Por ejemplo, 
en un foro académico que tuvo lugar en el 2017, un funcionario de una de las instituciones del Estado que se ocupa de la regulación de las prácticas agropecuarias en el país afirmaba:

Nosotros [refiriéndose a la entidad a la que representa] no tenemos nada en contra de las semillas nativas y criollas. Por el contrario, nos parece muy importante su conservación. Pero no podemos arriesgarnos a que un campesino que vaya caminando por ahí vea un cultivo que le parezca lindo, agarre la semilla y la siembre en su terreno sin saber ni siquiera de dónde venía esa semilla y con qué tipo de problemas, y luego terminemos metidos en un problema de plagas ni el tenaz. Por eso es que abogamos por las semillas certificadas, que ya están limpias y mejoradas para que los campesinos las usen con seguridad. (Servidor público, 2017, énfasis añadido)

Como puede verse en la cita anterior, aunque el servidor público sostiene que el Estado no tiene "nada en contra de semillas criollas y nativas", cuando continúa con su argumento demuestra que en realidad las considera riesgosas y posibles portadoras de plagas. Así mismo, subrayamos en la cita la forma en la que el funcionario imagina al campesino: como un ser ignorante que carece del conocimiento básico acerca de las semillas que selecciona para su cultivo. ¿Es este el caso de los campesinos colombianos? La forma en que el conocimiento se genera, comparte y materializa en las nuevas semillas criollas y en los alimentos de las redes de custodios de semillas que hacen parte de la RSLC muestra que, en efecto, tal imaginario dista mucho de la realidad.

A esta representación sobre las semillas nativas y criollas (y los campesinos) se suman los potenciales procesos de criminalización del campesinado creados por las mismas leyes de certificación y DPI. Para el caso de Colombia, como resultado del Tratado de Libre Comercio (TLC) con Estados Unidos que entró en vigor en el 2012, el país debió adherirse a la UPOV91 y, por tanto, cambiar su legislación de propiedad intelectual para penalizar el uso y la propagación de semillas no certificadas, así como limitar el derecho a resembrar semillas protegidas por DPI ${ }^{11}$.

La Resolución 3168 del Instituto Colombiano de Agricultura (ICA) ${ }^{12}$ y la Ley 1032 del 2006 que modifica el artículo 306 del Código Penal hacen parte de esta nueva legislación. La resolución del ICA establece que todas las semillas del país

Colombia ya había ratificado la UPOV desde 1996, pero en su versión de 1978. Esto es importante porque esta versión no restringe el intercambio libre de semillas, lo que sí hace explícitamente la versión de 1991. 
deben ser certificadas y que la semilla certificada solo puede ser resembrada una sola vez, en un área reducida y únicamente para el autoconsumo ${ }^{13}$. La modificación del Código Penal penaliza la usurpación de DPI con cuatro a ocho años de cárcel y multas entre 26 y 1.500 salarios mínimos legales vigentes (GóngoraMera y Motta 2014; Grupo Semillas 2011; Gutiérrez y Fitting 2016).

Como lo muestra nuestro análisis, el paradigma en el que se enmarcan tanto los procesos de patentes como de certificación de material vegetal parte de la idea de que la semilla debe ser estandarizada por medio de procedimientos tecnocientíficos. Esta concepción desconoce la diversidad biogenética y el carácter rápidamente adaptable de las llamadas semillas nativas y criollas a partir de la experimentación en los campos de cultivo de los agricultores, y les da primacía a las semillas intervenidas en laboratorios y campos experimentales controlados mediante técnicas de ingeniería genética (Fitting, Wattnem y Gutiérrez, en prensa).

Así las cosas, ¿qué sucede con el conocimiento de los guardianes de semillas? A continuación, profundizamos en este aspecto mostrando que la manera en que se concibe el conocimiento experto bajo la estructura de los DPI y las leyes de certificación de semillas opera como un mecanismo de exclusión de formas de saber no institucionalizadas.

\section{Cuidado de saberes y semillas}

La RSLC opera alrededor de tres ejes de trabajo: la conservación y la recuperación de las semillas a partir de capacitación e investigación propia, la divulgación de la problemática de las semillas y de las acciones de resistencia, y la incidencia política y el accionar jurídico (RSLC y Grupo Semillas 2015).

Si bien los tres ejes funcionan de manera articulada, en este artículo nos concentramos en el de conservación y recuperación de semillas nativas y criollas. En este eje ubicamos las escuelas agroecológicas, las redes regionales de guardianes de semillas, las ferias de semillas, las casas comunitarias de semillas y otras iniciativas que permiten a las comunidades y organizaciones de base conservar e intercambiar semillas nativas y criollas -y los saberes vinculados - en sus territorios, o lo que se conoce en la literatura especializada como conservación

fin, como resultado de las mesas de negociación entre organizaciones agrarias y el Gobierno nacional a raíz del Paro Agrario Nacional del 2013. 
in situ (RSLC y Grupo Semillas 2015). El componente de “capacitación” se refiere a un modelo de campesino a campesino (Holt-Giménez 2006) en conservación y recuperación de semillas criollas que utiliza técnicas y conocimientos locales combinados con prácticas agroecológicas. La particularidad de este modelo es que, como su nombre lo indica, los mismos campesinos son quienes se encargan de diseñar y formular los procesos de capacitación y circulación de saberes. Con ello se evade el enfoque desarrollista que privilegia el conocimiento experto certificado sobre el conocimiento situado. Para la RSLC los expertos existen pero no necesitan de credenciales universitarias que así lo indiquen, pues los expertos también son los campesinos.

Las redes de semillas de la RSLC generalmente promueven la agroecología en los territorios para producir semillas orgánicas en huertos y campos. También realizan inventarios participativos de semillas para determinar cuáles variedades son las más amenazadas y necesitan ser guardadas, reproducidas u obtenidas prioritariamente a través del intercambio con otras redes. Para fines educativos, las redes de guardianes de semillas publican catálogos que explican las características básicas de las semillas que están conservando.

Todos estos procesos son motivados en parte por los encuentros nacionales de guardianes de semillas, como el ocurrido en el Instituto Mayor Campesino (IMCA) en Buga en el 2017. Allí, distintos campesinos provenientes de todas las esquinas del país hicieron demostraciones de sus procesos de producción de semillas y de investigación de variedades nativas y criollas de hortalizas, que han realizado colectivamente durante meses e incluso años. Algunos asuntos básicos que se discutieron fueron: 1) cómo cada comunidad cuida de las semillas en invernaderos; 2) cómo maneja cada comunidad los germinadores y los procesos de plantulación con métodos agroecológicos; y 3) qué técnicas manuales y libres de agroquímicos se utilizan para la extracción, secado, limpieza y almacenamiento de semillas en cada red de custodios.

Algunas redes de semillas, como la apoyada por la Recab en el departamento de Antioquia, están trabajando en un registro documental de historias de vida de semillas basadas en testimonios orales de los mayores. Durante una feria nacional de semillas realizada en septiembre del 2014, Alejandro, un guardián de semillas de Antioquia, explicó que este registro tiene la intención de "reconstruir esa historia de cómo se prepararon los alimentos y se sembraron semillas; es como una arqueología del conocimiento”.

En el trabajo de campo realizado por Laura Gutiérrez con los guardianes de semillas de la organización de base Asproinca, provenientes de los resguardos de Riosucio (Caldas), se realizó un inventario de la agrobiodiversidad de las 
parcelas a partir de matrices de semillas. La tabla 1 muestra los múltiples conocimientos sobre plantas en los sistemas agroecológicos que promueve Asproinca, desde la alimentación de animales y humanos hasta métodos de control biológico que reemplazan parcialmente el uso de pesticidas y herbicidas a base de sustancias químicas.

Tabla 1. Usos de plantas en sistemas agroecológicos

\begin{tabular}{|c|c|}
\hline Plantas para forraje & $\begin{array}{l}\text { Bore, nacedero, botón de oro braquearia, matarratón, } \\
\text { caña brava. }\end{array}$ \\
\hline Plantas medicinales & $\begin{array}{l}\text { Prontoalivio, albahaca, romero, cilantro cimarrón, } \\
\text { mejorana, árnica, cidrón y ruda. }\end{array}$ \\
\hline $\begin{array}{l}\text { Plantas para control biológico y } \\
\text { conservación de semillas }\end{array}$ & Ají, ajo, salvia, artemisa, barbasco, ruda e higuerilla. \\
\hline Leguminosas para cuidado del suelo & $\begin{array}{l}\text { Guandul, teofrosia, crotalaria, churito, múcuna y } \\
\text { canabalia. }\end{array}$ \\
\hline $\begin{array}{l}\text { Plantas para descontaminación de } \\
\text { aguas residuales }\end{array}$ & Lechuguilla. \\
\hline $\begin{array}{l}\text { Plantas para procesamiento de } \\
\text { alimento }\end{array}$ & $\begin{array}{l}\text { Cadillo para limpiar el guarapo o bebida fermentada de } \\
\text { caña. }\end{array}$ \\
\hline
\end{tabular}

Fuente: Gutiérrez (2016, 302).

La tabla 2 consigna el conocimiento tradicional sobre algunas variedades de fríjol criollo que conservan las familias de Asproinca.

Tabla 2. Conocimiento tradicional sobre variedades de fríjol*

\begin{tabular}{l|l|l}
\multicolumn{1}{c|}{ Fríjol } & \multicolumn{1}{c}{ Color } & \multicolumn{1}{c}{ Características } \\
Cachas & $\begin{array}{l}\text { Rojas y negras con manchas } \\
\text { blancas }\end{array}$ & Resistente a los insectos. \\
\hline Fríjol de año & $\begin{array}{l}\text { Rosado, blanco, rojo y con rayas } \\
\text { marrones y blancas }\end{array}$ & $\begin{array}{l}\text { Capacidad de producir todo el } \\
\text { año. }\end{array}$ \\
\hline Bugueño & Marrón y blanco & $\begin{array}{l}\text { Apreciado por su sabor. Su nom- } \\
\text { bre denota que probablemente } \\
\text { fue traído desde Buga, en el } \\
\text { Valle del Cauca. }\end{array}$ \\
\hline Cuero de vieja & Negra, amarilla y marrón & $\begin{array}{l}\text { Su nombre se refiere a la piel } \\
\text { arrugada y dura del fríjol, que } \\
\text { parece la piel de una anciana. }\end{array}$
\end{tabular}




\begin{tabular}{l|l|l}
\multicolumn{1}{c|}{ Fríjol } & \multicolumn{1}{c|}{ Color } & \multicolumn{1}{c}{ Características } \\
Petaco & Marrón & $\begin{array}{l}\text { Crece en las tierras altas y frías } \\
\text { y produce durante todo el año. }\end{array}$ \\
\hline Algarrobo & Azuloso con rayas blancas & $\begin{array}{l}\text { Es un fríjol arbustivo, escaso y } \\
\text { apreciado por su sabor. }\end{array}$ \\
\hline
\end{tabular}

* Todas las variedades son de enredadera, a menos que se indique lo contrario.

Fuente: Gutiérrez (2016, 311).

Estas iniciativas buscan promover la soberanía alimentaria y en semillas. El movimiento transnacional La Vía Campesina (2007) ha definido la soberanía alimentaria como el derecho de todos los pueblos y naciones a determinar sus sistemas alimentarios de manera social y ambientalmente justa y sostenible, sin estar supeditados a corporaciones alimentarias, organizaciones multilaterales y al sistema financiero mundial. La soberanía alimentaria implica la defensa de los conocimientos y prácticas agroalimentarias y los territorios de los pueblos productores de alimentos (Gutiérrez 2018; LVC 2007). Partiendo de este concepto, la soberanía en semillas plantea el derecho de los agricultores, pueblos y países a decidir autónomamente sobre sus sistemas de semillas. Dichas decisiones se toman acerca de las cualidades y tipos de semillas, así como sobre las formas en que son cultivadas, guardadas y circuladas, de manera que cumplan con expectativas y necesidades agroecológicas, culturales y productivas de los territorios (Kloppenburg 2010).

\section{Habitar, saber y semilla}

Las resistencias epistémico-políticas de la RSLC también incluyen la construcción de materialidades e infraestructuras que abren espacios para la creación de comunidad y el intercambio de saberes y de semillas, como las casas comunitarias de semillas (CCS). Allí, los guardianes de semillas aprenden y enseñan a cuidar, reproducir y distribuir semillas criollas y nativas a través de talleres, donaciones, trueque y venta. A continuación, nos centramos en la Casa de Semillas del Resguardo Embera-Chamí de Cañamomo y Lomaprieta. Esta se articula con la declaración del resguardo como territorio libre de transgénicos (TLT) o la prohibición -basada en la autonomía indígena- de cultivar y consumir variedades transgénicas; prohibición que incluye a entidades gubernamentales y privadas, como las ONG que promuevan programas de seguridad alimentaria y de 
desarrollo agropecuario ${ }^{14}$. Esta prohibición también se sustenta en las medidas de bioseguridad adoptadas por el ICA que prohíben la siembra de variedades transgénicas de maíz en resguardos indígenas y a menos de 300 metros de estos. En la declaración de TLT también hay un compromiso para la defensa de las semillas nativas y criollas y los conocimientos tradicionales asociados.

La casa fue construida en una finca que hace parte de los terrenos comunitarios del resguardo, en los que cualquiera puede sembrar y recoger frutos, siempre y cuando haga parte de la comunidad y cumpla con tres condiciones básicas: 1) usar semillas criollas y nativas o comerciales si son significativas a nivel económico; 2) no utilizar paquetes tecnológicos del ICA —o cualquier otra entidad gubernamental o privada- que estén basados en agroquímicos o variedades transgénicas que agredan a las semillas, a los demás guardianes y cultivadores ni a los demás organismos vivos del lugar; y 3) estar dispuesto a compartir sus saberes y productos con los demás miembros del resguardo, de la RSLC y otras comunidades, y rechazar cualquier forma de propiedad intelectual.

La Casa de Semillas es pequeña y está construida con fique y otros materiales de la zona. Tiene espacios dedicados al almacenamiento de semillas, que antes de ingresar en los pequeños contenedores de vidrio son seleccionadas y clasificadas. Así mismo, la casa abre sus puertas para recibir a los guardianes que se reúnen, no solo para intercambiar semillas, sino también para discutir acerca de las dificultades o bondades de determinados cultivos e insumos agroecológicos utilizados, compartir los resultados e ideas de procesos bioexperimentales y, por supuesto, contar anécdotas y compartir la vida. Así, se convierte en un espacio en el que saber y ser (colectivo) van de la mano.

La Campaña de Semillas de Identidad, de la agencia de cooperación suiza SwissAid, ha trabajado con la Casa de Semillas para diseñar e implementar "registros de semillas” o pasaportes de semillas sobre las semillas conservadas y sus guardianes. Hay un pasaporte para cada variedad conservada que registra su nombre científico y local y lo que significa, su origen o el lugar, la persona de quien se adquirió y la fecha, la familia o guardián local de semillas que se ocupan de conservarla, su descripción botánica, sus beneficios (alta en nitrógeno, muy sabrosa, etc.), las mejores técnicas de crecimiento y conservación, y

14 Las zonas o territorios libres de transgénicos han aparecido alrededor del mundo como una forma de protección socioambiental frente al uso de semillas transgénicas. Son zonas en las que, por ley, la siembra de cultivos transgénicos no está autorizada y se le da prioridad al uso de semillas nativas y criollas o, como las llaman en otros países, semillas campesinas. Según el grupo español Ecologistas en Acción, en el 2006, 170 regiones europeas se declararon TLT. A estas declaraciones siguieron numerosas en África y América Latina. SwissAid Colombia estima que actualmente existen siete TLT en nuestro país, la mayoría en territorios indígenas. 
sus usos alimentarios, medicinales, agronómicos y culturales. De esta forma, la Casa de Semillas sabe dónde buscar los suministros de semillas y se asegura de que no quede ninguna sin ser asignada a un guardián - particularmente aquellas que están en peligro de extinguirse y necesitan ser reproducidas y cuidadas sistemáticamente-.

De igual manera, la Casa de Semillas se compromete con la resistencia epistémica al rechazar la certificación del ICA basada en criterios que incluyen principalmente la productividad, la pureza y la homogeneidad genética; criterios que solo las semillas de las empresas - ya sean híbridas o transgénicas- pueden cumplir y que clasifican a las semillas criollas como improductivas y riesgosas en términos de estándares fitosanitarios (tabla 3). Para los guardianes de semillas de Riosucio - y, en general, los guardianes de semillas de la RSLC-, las semillas certificadas se producen masivamente, en ecosistemas diferentes de aquellos de los agricultores, son muy susceptibles al clima y a condiciones de suelos pobres, que a menudo son característicos de la agricultura campesina e indígena, y solo funcionan bien en combinación con costosos paquetes tecnológicos.

Tabla 3. Acosemillas: semillas autorizadas y no autorizadas por el ICA

\begin{tabular}{c|c}
$\begin{array}{c}\text { SEMILLA AUTORIZADA } \\
\text { POR EL ICA } \\
\text { (Alta calidad y costos racionales) }\end{array}$ & $\begin{array}{c}\text { SEMILLA NO AUTORIZADA } \\
\text { POR EL ICA }\end{array}$ \\
Expresión de características genéticas estables & (Baja calidad y costos elevados) \\
\hline Libre de plagas y enfermedades (inocuidad) & Transmisión de plagas y enfermedades \\
\hline Semilla con alto porcentaje de pureza & Presencia de material inerte y semillas no aptas \\
para el cultivo
\end{tabular}

Fuente: Elaboración propia a partir de http://www.acosemillas.com/noticia-3 / Consultado en noviembre del 2017. 
Durante un taller, los socios de Asproinca en Riosucio y el municipio vecino de Supía expresaron sus consideraciones sobre las semillas criollas y certificadas. Según el testimonio de Hernando, un socio de Supía:

En el Comité de Cafeteros una vez dieron semilla certificada de fríjol y de maíz. Yo la sembré en un clima muy bueno, que es en la parte de Matecaña, y no me nació ni pa comer, y el maíz lo mismo. Y sembré de ese tradicional, del de nosotros, y eso dio unas mazorcas muy buenas. Esa semilla [certificada] viene atada para que usted enseguida traiga el bulto de abono. (Jaime, diario de campo, octubre del 2013)

Además, los guardianes rechazan las semillas certificadas y los procesos de certificación porque los perciben como una herramienta para descalificar y desestimular el uso de semillas nativas y criollas y el desarrollo de métodos locales para su cultivo y adaptabilidad. Por ejemplo, en una reunión de guardianes de semillas y miembros de los cabildos de Riosucio en el 2014, se discutió el conflicto por el requisito de utilizar la semilla certificada del ICA en los programas de seguridad alimentaria de la Red de Seguridad Alimentaria o ReSa del Ministerio de Agricultura y Desarrollo Rural. Las autoridades indígenas insistieron en condicionar la implementación de ReSa en los resguardos de Riosucio al uso de semillas criollas, no certificadas por el ICA, y provenientes de la Casa de Semillas o las redes de guardianes de semillas. En la reunión, Rosa, una guardiana de semillas, recordó con orgullo cómo desafiaron al personal de ReSa diciendo: "lo sentimos, pero aquí tenemos nuestra propia propuesta, nuestras propias semillas, y no queremos semillas certificadas”.

En consecuencia, la Casa de Semillas ha desarrollado sus propios estándares de calidad al crear un Sistema Participativo de Garantías (PGS). El PGS se basa principalmente en el conocimiento y la experimentación de los agricultores para el desarrollo de variedades heterogéneas en sus parcelas, aunque incorporando elementos de "la ciencia occidental", cuando lo consideran apropiado, en un ejercicio de diálogo de saberes. La Casa de Semillas requiere suministrar semillas adaptadas a las condiciones locales y cultivadas y reproducidas sin el uso de agroquímicos, aunque también utiliza estándares convencionales que miden, por ejemplo, porcentajes de germinación o de humedad de las semillas. Además se emplean distintas técnicas de conservación de forma simultánea: "la compra de refrigeradores para el almacenamiento está en consideración, pero ají y otras plantas tradicionales se utilizan para prevenir la infestación por gorgojos y otros insectos”, dice Rosa.

Como se ve en la tabla 3, Acosemillas, la asociación de empresas semilleras nacionales y transnacionales que operan en el país, contrapone las semillas 
autorizadas y no autorizadas por el ICA en términos de competitividad y sostenibilidad que garantizan el "éxito o fracaso" de los cultivos. Como explicamos en la sección "Leyes de semillas", dados los criterios de productividad, pureza y homogeneidad genética, el ICA termina certificando solamente las semillas de las empresas que son las que pueden asumir los costos de certificación. Por tanto, se puede inferir que las semillas no certificadas a las que se refiere la tabla 3 son semillas criollas que no pueden cumplir con los requisitos de certificación.

La definición de lo que es una "buena” semilla difiere marcadamente entre los criterios del ICA/Acosemillas y los de los guardianes de semillas que se observan en la tabla 4. Para el ICA/Acosemillas, la calidad de las semillas se expresa en su productividad y competitividad, medida en términos de germinación, pureza, estabilidad, rendimiento, inocuidad y menores costos de producción. Si bien la evaluación de la calidad de las semillas por parte de la RSLC comparte algunos criterios con la del ICA/Acosemillas - como la inocuidad, pero entendida en términos de mayor adaptabilidad a enfermedades y plagas-, existen otros criterios muy distintos y propios de las economías campesinas y de otros modelos alternativos, como la agroecología, que plantea sistemas productivos que "son biodiversos, resilientes, eficientes energéticamente, socialmente justos y constituyen la base de una estrategia energética y productiva fuertemente vinculada a la soberanía alimentaria” (Altieri y Toledo 2010, 4). En este sentido, la RSLC propone sistemas de mejoramiento y propiedad de las semillas que promuevan la heterogeneidad genética y biológica, para permitir la adaptabilidad de las semillas a diversas condiciones ecosistémicas, culturales o productivas, en especial a los cultivos diversificados y a las tradiciones de libre desarrollo e intercambio.

Tabla 4. Semillas nativas y criollas versus semillas certificadas (híbridas y transgénicas)

\begin{tabular}{|c|c|}
\hline Semillas nativas y criollas & $\begin{array}{l}\text { Semillas certificadas } \\
\text { (híbridas y transgénicas) }\end{array}$ \\
\hline Bienes comunes; patrimonio de los pueblos & $\begin{array}{l}\text { Propiedad privada de las empresas por propiedad } \\
\text { intelectual; monopolio }\end{array}$ \\
\hline Mejoramiento genético descentralizado y colectivo & $\begin{array}{l}\text { Mejoramiento genético centralizado y } \\
\text { descontextualizado }\end{array}$ \\
\hline Heterogeneidad (alta diversidad de variedades) & Homogeneización (baja diversidad de variedades) \\
\hline $\begin{array}{l}\text { Adaptadas a condiciones ambientales, culturales y } \\
\text { productivas }\end{array}$ & Ambientes controlados e insumos externos \\
\hline Sistemas diversificados/agroecológicos & Monocultivo agroindustrial \\
\hline
\end{tabular}




\section{Semillas nativas y criollas}

\begin{tabular}{|c|c|}
\hline Semillas nativas y criollas & $\begin{array}{l}\text { Semillas certificadas } \\
\text { (híbridas y transgénicas) }\end{array}$ \\
\hline Mayor adaptación a sequía, enfermedades y plagas & Más susceptibles a sequía, enfermedades y plagas \\
\hline $\begin{array}{l}\text { Flexibilidad para cambio climático y mitigación del } \\
\text { hambre }\end{array}$ & $\begin{array}{l}\text { Ambientes limitados y no diseñadas para mitigar } \\
\text { el hambre }\end{array}$ \\
\hline $\begin{array}{l}\text { Más nutritivas y sanas, ya que en su mayoría son } \\
\text { cultivadas sin agroquímicos y consumidas sin } \\
\text { mayor procesamiento }\end{array}$ & $\begin{array}{l}\text { Se cultivan con un alto uso de agroquímicos y } \\
\text { son el insumo básico de la industria de alimentos } \\
\text { ultraprocesados }\end{array}$ \\
\hline
\end{tabular}

Fuente: elaboración propia a partir de RSLC $(2015,17)$.

\section{Desafíos de la defensa de las semillas como bien común y la descolonización de los saberes y prácticas sobre agrobiodiversidad}

Como toda iniciativa popular, la Red de Semillas Libres de Colombia enfrenta desafíos, evidenciados durante nuestro trabajo de campo. Algunos de los retos mencionados tienen que ver con la forma en la que el mercado ha transformado las preferencias y los hábitos alimentarios del consumidor. Al respecto, Mariana, una guardiana de semillas de Bogotá, comentaba:

Es difícil para nosotros sacar nuestros productos al mercado, hacer que la gente los compre y los consuma. A la gente le gustan las frutitas y verduras bien parejitas, de vitrina. Las nuestras son más feítas; son chiquitas y medio deformes [risas]. Aparte, muchos de los productos que sacamos al mercado y tratamos de vender ni los conocen. O si los conocen, no les gustan. Mire, por ejemplo, acá mismo en Bogotá es muy difícil vender cubios e hibias. Los ven como comida de pobre. Prefieren sus productos importados o los dos tubérculos que siempre han comido. (Entrevista, 2017)

El comentario de Mariana es un ejemplo de cómo la expansión de las cadenas alimentarias transnacionales, junto con la importación y la industrialización de la producción de alimentos que imponen la homogeneización y estandarización, contribuyen a la marginación de las variedades nativas y criollas en los mercados (Coomes et al. 2015). También muestra de qué manera los mercados crean y reflejan estructuras racializadas y de clase en las que ciertas comidas son 
asociadas con los “pobres", indios o negros y, por tanto, despreciadas. Igualmente, el cultivo de semillas criollas implica cultivar una forma no hegemónica de estética - una aesthesis decolonial (Vásquez y Mignolo 2013) ${ }^{15}$ — en la que las nociones de "belleza" — que, a su vez, están asociadas a la calidad- no necesariamente se relacionan con la homogeneidad, la "perfección” y el tamaño grande; más bien cada fruto y semilla es perfecto y hermoso en su diversidad y heterogeneidad ${ }^{16}$.

La pérdida de los saberes sobre la siembra y conservación de variedades criollas también es un riesgo presente por la falta de relevo generacional, dada la alta migración de jóvenes a las zonas urbanas, las minas, la agroindustria o los cultivos de uso ilícito, en busca de “mejores oportunidades”. Puesto que los jóvenes no ven en el saber de sus padres y abuelos ningún uso que se asocie a la generación de condiciones de vida más dignas (en términos materiales o sociales), se desplazan hacia centros donde el flujo de efectivo es mayor y más frecuente.

Otro desafío es la coordinación del suministro de semillas para abastecer la demanda local y regional, de tal manera que circulen fluidamente. Al respecto, Rosa explicó que "a veces tenemos muchas solicitudes y no tenemos suficiente semilla. A veces, las semillas permanecen almacenadas por mucho tiempo y pueden dañarse”. La escasez de semilla es especialmente aguda cuando la Casa de Semillas necesita abastecer programas públicos y privados de ayuda y seguridad alimentaria y de desarrollo agrícola. Por ejemplo, Manuel, un guardián de Cañamomo y Lomaprieta quien también trabaja con el gobierno municipal, arguyó en una reunión en mayo del 2014 que:

Hemos tenido dificultades para reemplazar la Bienestarina [un suplemento alimenticio] por guineo [una variedad criolla de plátano] o por colada de maíz criollo porque no tenemos los cultivos. Hay pocos cosecheros de maíz y plátano. La mayor parte del maíz se cultiva en las tierras frías donde el ciclo es más largo —entre ocho y nueve mesesmientras que no cultivamos en las tierras calientes, donde el ciclo es más corto, porque en su mayor parte hay caña.

15 Para Vásquez y Mignolo (2013), todos los seres sintientes, incluidos los humanos a lo largo de la evolución y la historia, han desarrollado sus propias formas de relacionarse con lo sensible, de "percibir lo bello y lo sublime" o aestheSis. Por lo tanto, los autores usan el término aestheTics para referirse a la forma de regulación de los sentidos que surgió en la Europa del siglo XVIII, particularmente en el trabajo de Immanuel Kant, y se convirtió en el "regulador de la capacidad global" para percibir "lo bello y lo sublime". A medida que las estéticas (aesthetics) se incorporaron a la matriz colonial del poder, comenzaron a colonizar las estéticas-otras (aesthesis) de los pueblos no occidentales. En otras palabras, a borrar y denigrar otras formas de percepción, así como a imponer sensibilidades eurocentradas como universales y superiores.

Por ejemplo, aunque los guardianes de semillas pueden seleccionar los granos más grandes en una variedad para la próxima cosecha, no descartan pequeñas variedades porque pueden ser importantes en términos nutricionales, agronómicos o culturales. 
Así mismo, la amenaza de que el Gobierno implemente nuevas leyes que criminalicen la libre reproducción, circulación y comercialización de semillas, incluidas las variedades criollas, no ha desaparecido con los recientes logros de la RSLC en materia legal. En diciembre del 2012, después de una demanda de la $\operatorname{Red}^{17}$, la Corte Constitucional declaró inexequible la Ley 1518 que aprobaba la UPOV91. Sin embargo, las legislaciones que implementa la UPOV91, como la Resolución 3168 del ICA o la Ley 1032 del 2006, siguen vigentes puesto que la sentencia de la Corte no se pronuncia sobre ellas. Esto podría significar la confiscación y destrucción de semillas, así como la aplicación de multas y la judicialización de los encargados de la Casa de Semillas por el uso y reproducción de semillas criollas que no están certificadas por el ICA o por la violación de derechos de propiedad intelectual de semillas certificadas. Si bien los resguardos indígenas cuentan con los derechos de autonomía y gobierno propio garantizados por la Constitución, no es extraño en Colombia que las autoridades estatales los violen.

Finalmente, la Casa de Semillas y los guardianes también temen la contaminación genética de las variedades criollas debido a que las variedades transgénicas entran en la cadena agroalimentaria vía el maíz importado de Estados Unidos, Argentina y otros países. También, porque el Gobierno, los organismos multilaterales y algunas ONG usan variedades transgénicas en sus programas de seguridad y ayuda alimentaria y desarrollo agrícola, dado que las consideran más productivas y pueden adquirirlas más baratas en el mercado. Por ejemplo, el Gobierno central promueve el Plan Maíz-País que busca impulsar este cultivo entre pequeños cultivadores para la producción de concentrado para la industria del cerdo y la avicultura (Fenalce 2011, 5). Sin embargo, los agricultores que deseen obtener beneficios del Plan Maíz-País deben utilizar semillas de maíz, transgénico o híbrido, certificadas por el ICA. Esto no solo afecta la economía local del maíz criollo y los programas de soberanía alimentaria en territorios como los resguardos de Riosucio, sino que plantea la amenaza de contaminación genética de las variedades criollas debido a la polinización cruzada. La contaminación genética representa una amenaza tanto para la preservación de las semillas criollas y nativas como para los conocimientos de los guardianes de semillas. No obstante, al mismo tiempo, es gracias al conocimiento profundo de las semillas y el medio ambiente que muchos casos de contaminación genética han sido detectados en comunidades y luego confirmados usando alianzas estratégicas con científicos independientes aliados de la Red. 


\section{Continuar caminando las semillas}

Comenzamos este artículo preguntando qué es lo que está en conflicto cuando hablamos de los conflictos por las semillas en Colombia y qué formas específicas toman dichos conflictos en nuestro país. En el recorrido realizado mostramos que, aunque lo que está en conflicto pasa de manera esencial por el uso y la circulación de las semillas, resulta fundamental estudiar también los problemas generados alrededor de los conocimientos acerca de su cultivo, conservación, posibles procesos de hibridación y mejoramiento, entre otros. En otras palabras, hemos querido resaltar la importancia de analizar la dimensión epistémica de los conflictos sobre las semillas dado que estas no pueden ser reducidas a un "recurso" o medio de producción, sino que son parte integral del mundo de millones de campesinos, indígenas y afrolatinos en nuestro continente. Centrándonos en el eje de conservación, recuperación y circulación de semillas de la RSLC, mostramos cómo para esta organización saber y semilla son dos elementos inseparables, arraigados al territorio y las comunidades.

Las formas de saber tecnocientíficas y la estructura jurídico-política que regula a la industria biotecnológica, y que es promovida por organismos estatales, se basan en dos premisas de la colonialidad del saber (Mignolo 2002) — que es parte integral de la colonialidad cultural-. Primero, que existe una única forma de conocimiento sobre el mundo natural que puede ser legitimada a la hora de producir política pública sobre las semillas. Segundo, que dicha forma de conocimiento puede ser retribuida económicamente solo cuando se encuentra como propiedad privada, esta vez determinada bajo la forma de la propiedad intelectual.

Frente a esta estructura de dominación caracterizada por la colonialidad cultural y del saber, la respuesta del giro decolonial es clara: los proyectos de carácter decolonial deben hacerse cargo de producir, generar y dar cuenta de un "saber otro" que nace de la diferencia colonial (y que reconoce a sus participantes desde sus distintas espacialidades geográficas, corporales y socioculturales), pero sin "reproducir la visión universalista eurocéntrica ni pretender la neutralidad de saberes” (Mignolo 2003, 27).

Uno de nuestros argumentos principales es que el trabajo que realiza la RSLC se enmarca dentro del contexto decolonizador, puesto que sus formas de resistencia no se basan únicamente en la movilización social, cuya importancia es fundamental, sino también en la construcción y el mantenimiento de una cultura de producción y circulación de un saber-ser-hacer cuya determinación epistémica es también política. Ese conocimiento propio de los pueblos indígenas, campesinos y afrocolombianos muestra que la semilla es un material orgánico, 
sujeto de conocimiento y representación del pasado, el presente y el futuro de los pueblos que luchan para mantenerla libre y en movimiento.

De esta manera, la RSLC desafía el poder de las corporaciones de biotecnología, dado que las semillas no son consideradas mercancías para la acumulación de capital protegidas por DPI. Si bien la circulación y comercialización de semillas criollas y nativas es demasiado baja para afectar económicamente el mercado corporativo, la RSLC sí ha tenido un impacto político que reivindica el derecho a la soberanía en semillas -incluyendo formas propias de saber sobre ellas-y el cuestionamiento a su cercamiento corporativo. Muestra de esto son las demandas legales contra las leyes de semillas basadas en la UPOV91 y las iniciativas de conservación y defensa de variedades criollas por medio de redes de guardianes de semillas y casas comunitarias de semillas.

Queremos entonces enfatizar que es fundamentalmente gracias al arduo trabajo que los guardianes de semillas realizan a lo largo y ancho del país, que cientos de variedades de semillas criollas y nativas, y los saberes asociados con su siembra, germinación y consumo, continúan caminando libremente.

\section{Referencias}

Altieri, Miguel y Víctor Toledo. 2010. La revolución agroecológica en Latinoamérica. Bogotá: Socla.

Collins, Harry y Robert Evans. 2007. Rethinking Expertise. Chicago: The University of Chicago Press.

Coomes, Oliver T., Shawn J. McGuire, Eric Garin, Sophie Caillon, Doyle McKey, Elise Demeulenaere, Devra Jarvis, Guntra Aistara, Adeline Barnaud, Pascal Clouvel, Laure Emperaire, Sélim Louafi, Pierre Martin, François Massol, Marco Pautasso, Chloé Violon y Jean Wencélius. 2015. "Farmer Seed Networks Make a Limited Contribution to Agriculture? Four Common Misconceptions”. Food Policy 56: 41-50. https://doi.org/10.1016/j. foodpol.2015.07.008.

Corrales, Elcy y Jaime Forero. 2007. La reconstrucción de los sistemas de producción campesinos: el caso de Asproinca en Riosucio y Supía. Bogotá: Pontificia Universidad Javeriana.

Epstein, Steve. 1996. Impure Science: Aids, Activism, and the Politics of Knowledge. Berkeley; Los Ángeles: University of California Press.

Escobar, Arturo. 2011. Encountering Development: The Making and Unmaking of the Third World. Princeton: Princeton University Press.

Fenalce. 2011. "Plan Maíz-País”. El Cerealista (enero-marzo). http://www.fenalce.org/arch_ public/paismaiz96.pdf 
Fitting, Elizabeth, Tamara Wattnem y Laura Gutiérrez Escobar. (En prensa). "Contesting Seed Standards: The Red de Semillas Libres in Colombia”. En Localizing Standards, editado por Christina Holmes y Janice Graham. Vancouver: University of British Columbia Press.

Gobierno Nacional de Colombia. 2006. "Tratado de Libre Comercio con Estados Unidos: Acuerdo para la Promoción Comercial entre la República de Colombia y Estados Unidos de América”. Consultado el 1. ${ }^{\circ}$ de marzo del 2018. http://www.tlc.gov.co/publicaciones/14853/ acuerdo_de_promocion_comercial_entre_la_republica_de_colombia_y_estados_unidos_ de_america

Góngora-Mera, Manuel y Renata Motta. 2014. "El derecho internacional y la mercantilización biohegemónica de la naturaleza: la diseminación normativa de la propiedad intelectual sobre semillas en Colombia y Argentina”. En Desigualdades socioambientales en América Latina, editado por Bárbara Göbel, Manuel Góngora-Mera y Astrid Ulloa, 395-434. Bogotá; Berlín: Universidad Nacional de Colombia; Ibero-Amerikanisches Institut.

Grupo Semillas. 2011. Las leyes de semillas aniquilan la soberanía y autonomía de los pueblos. Bogotá: Arfo Editores.

Gutiérrez Escobar, Laura. 2016. "The Political Ontology of Seeds: Seed Sovereignty Struggles in an Indigenous Resguardo in Colombia”. Tesis doctoral, University of North Carolina at Chapel Hill.

-. 2018. "Food Sovereignty and Autonomy". En The Postdevelopment Dictionary. A Guide to the Pluriverse, editado por Ashis Kothari, Federico Demaria, Alberto Acosta, Ariel Salleh y Arturo Escobar 185-188. Londres: Zed Books.

Gutiérrez Escobar, Laura y Elizabeth Fitting. 2016. “The Red de Semillas Libres: Contesting Biohegemony in Colombia”. Journal of Agrarian Change 16 (4): 711-719. https://doi. org/10.1111/joac.12161

Harvey, David. 2004. El nuevo imperialismo: acumulación por desposesión. Buenos Aires: Clacso.

Holt-Giménez, Eric. 2006. Campesino a Campesino, Voices from Latin America's Farmer to Farmer Movement. Oakland: Food First Books.

Jasanoff, Sheila. 1990. The Fifth Branch: Science Advisers as Policy Makers. Cambridge, MA: Harvard University Press.

- 2013. "Technologies of Humility: Citizen Participation in Governing Science”. Minerva 41: 223-244. https://doi.org/10.1023/A:1025557512320

Kinchy, Abby J. 2012. Seeds, Science, and Struggle: The Global Politics of Transgenic Crops. Cambridge: The MIT Press.

Kloppenburg, Jack. 2005. First the Seed: The Political Economy of Plant Biotechnology, 14922000, 2. ${ }^{\text {a }}$ d. Nueva York: Cambridge University Press.

-. 2010. "Seed Sovereignty and the Promise of Open Source Biology". En Food Sovereignty. Reconnecting Food, Nature and Community, editado por Nettie Wiebe, Hannah Wittman y Annette Aurélie Desmarais, 152-167. Halifax; Winnipeg: Fernwood.

(LVC) La Vía Campesina. 2007. Declaración de Nyéleni. Consultado el 23 de noviembre del 2017. https://nyeleni.org/spip.php?article291 
Maldonado-Torres, Nelson. 2007. "Sobre la colonialidad del ser: contribuciones al desarrollo de un concepto”. En El giro decolonial: reflexiones para una diversidad epistémica más allá del capitalismo global, editado por Ramón Grosfoguel y Santiago Castro-Gómez, 127168. Bogotá: Pontificia Universidad Javeriana.

Mignolo, Walter. 2002. "The Geopolitics of Knowledge and the Colonial Difference”. The South Atlantic Quarterly 101 (1): 57-96. https://doi.org/10.1215/00382876-101-1-57

-. 2003. "Globalization and the Geopolitics of Knowledge: The Role of the Humanities in the Corporate University”. Nepantla: Views from South 4 (1): 97-119.

Nazarea, Virginia. 1995. Local Knowledge and Agricultural Decision Making in the Philippines: Class, Gender, and Resistance. Ithaca, NY: Cornell University Press.

Parthasarathy, Shobita. 2017. Patent Politics: Life Forms, Markets, and the Public Interest in the United States and Europe. Chicago: Chicago University Press.

Quijano, Aníbal. 2007. “Coloniality and Modernity/Rationality”. Cultural Studies 21: 168-178. https://doi.org/10.1080/09502380601164353

RSLC (Red de Semillas Libres de Colombia) y Grupo Semillas. 2015. Las semillas patrimonio de los pueblos al servicio de la humanidad: memorias de los encuentros nacionales de la Red Semillas Libres de Colombia. Bogotá: RSL; Grupo Semillas.

Shiva, Vandana. 2001. Protect or Plunder? Understanding Intellectual Property Rights. Londres: White Lotus.

Vásquez, Rolando y Walter Mignolo. 2013. "Decolonial AestheSis: Colonial Wounds/Decolonial Healings”. Social Text Online. Consultado el 2 de abril del 2018. https://socialtextjournal.org/periscope_article/decolonial-aesthesis-colonial-woundsdecolonial-healings/ 